\title{
Parasitisme interne du mouton de race Ouled Djellal en zone semi-aride d'Algérie
}

\author{
A. Boulkaboul ${ }^{1 *}$ K. Moulaye ${ }^{1}$
}

\begin{abstract}
Mots-clés
Ovin Ouled Djellal - Nematoda Coccidia - Cestoda - Fèces -

Zone semi-aride - Algérie.
\end{abstract}

\begin{abstract}
Résumé
Une étude du parasitisme interne a été réalisée chez des ovins de race Ouled Djellal infestés naturellement dans une région semi-aride d'Algérie (Tiaret). Des coproscopies effectuées pendant l'année 2004 sur 423 ovins ont révélé un taux global d'infestation de 78,9 p. 100 et ont permis d'identifier des cestodes (Moniezia spp., Avitellina sp.), des coccidies (Eimeria spp.) et des nématodes dont des strongles respiratoires (Dictyocaulus sp., Muellerius sp., Protostrongylus sp.). Le parasitisme a été saisonnier pour tous les parasites. Les coccidies ont été plus présentes chez les jeunes $(n=164)$, avec une prévalence de 44,5 p. 100. Chez les brebis $(n=240)$, I'infestation par les strongles digestifs a été la plus élevée (70,4 p. 100), avec un pic en mars et en novembre. Les strongles suivants ont été identifiés : Trichuris ovis (5,8 p. 100), Marshallagia marshalli (28,3 p. 100) et Nematodirus spp. (27 p. 100). Les autres strongles, dont certains genres/espèces ont été identifiés par coproculture, ont eu une prévalence de 56,6 p. 100. M. marshalli et Nematodirus spp. ont eu une évolution remarquablement similaire, mais d'un niveau plus faible que celle des autres strongles. L'excrétion des œufs de strongles par gramme de fèces (opg) a présenté deux pics dont le plus élevé (moyenne de 1081 opg) s'est produit en novembre (période d'agnelage), en raison, probablement, du phénomène d'augmentation des opg lors du péripartum. Globalement, le parasitisme lié aux strongles a été modéré, malgré sa prédominance. La prévalence des opg individuels inférieurs à 500 a été de 74,1 p. 100, celle des opg supérieurs ou égaux à 1000 n'a été que de 14 p. 100 et la moyenne mensuelle d'opg n'a dépassé ce seuil qu'en novembre. Outre les conditions climatiques, l'implication de certains facteurs, tels que la nature du couvert végétal de la région et la race ovine, mérite investigation. Pour la prophylaxie, deux traitements anthelminthiques au cours de l'année (printemps et automne) doivent être suffisants.
\end{abstract}

\section{INTRODUCTION}

Le parasitisme interne, largement connu chez le bétail, fait intervenir divers parasites à l'origine de pathologies endémiques, sources de pertes par le retard de croissance, la chute des productions en viande, en lait, en laine, et par la mortalité $(16,27)$.

En Algérie, les parasites internes des ruminants domestiques identifiés macroscopiquement sont essentiellement partagés entre des nématodes ( 22 genres), des cestodes ( 9 genres) et des trématodes (3 genres) (28).

\footnotetext{
1. Université Ibn Khaldoun, département des Sciences vétérinaires, Tiaret, Algérie * Auteur pour la correspondance

2, rue du vieux palais, Alger 16000, Algérie.

E-mail : b_boulkaboul@yahoo.fr
}

Le cheptel ovin domine l'élevage avec un effectif d'environ 18,7 millions de têtes (ministère de l'Agriculture, 2004), en majorité concentré dans la zone des hautes plaines steppiques du Sud, où la wilaya (province) de Tiaret représente un important carrefour. Le parasitisme interne y constitue un obstacle à son développement, d'autant plus que le suivi sanitaire et zootechnique est insuffisant. Dans cette région, les parasites en cause sont principalement des découvertes d'abattoir où ont été recensés Cysticercus tenuicolis, Echinococcus granulosus, Coenurus cerebralis, Fasciola hepatica, Sarcocystis sp. et Dictyocaulus filaria, alors que peu d'intérêt est porté aux autres parasites des appareils respiratoire et digestif (6).

Une enquête antérieure réalisée auprès de vétérinaires et d'éleveurs de la région a révélé que des traitements de routine sont effectués en général deux à trois fois par an, sans aucun contrôle coprologique préalable. Ce travail tente donc de mettre en lumière la situation réelle du parasitisme interne des ovins de cette région, relatif 
plus particulièrement aux strongles, qui permettrait notamment de rationaliser l'utilisation des anthelminthiques, afin de repousser tant que possible le développement d'une résistance inévitable chez les parasites (26), comme cela a été rapporté pour les strongles digestifs chez des chevaux de la région (7).

\section{MATERIEL ET METHODES}

\section{Zone d'étude}

La région de Tiaret en Algérie appartient à la zone des hauts-plateaux, intercalée entre l'Atlas tellien et l'Atlas saharien, à une altitude moyenne de $1000 \mathrm{~m}$. C'est une région semi-aride, à vocation agropastorale, caractérisée par la céréaliculture et par un élevage ovin dominant. Ce dernier compte un cheptel de 800000 têtes, principalement de races Ouled Djellal, Hamra et Rumbi (15). Le climat y est rigoureux, avec une saison hivernale courte et froide, une saison chaude longue et sèche, ainsi qu'une pluviométrie limitée, de l'ordre de 350 à $400 \mathrm{~mm} / \mathrm{an}$ (figure 1).

\section{Animaux}

L'étude a concerné des ovins de race Ouled Djellal, excellente race à viande, adaptée aux zones arides (15), dominante dans la région. Les éleveurs pratiquent un élevage extensif à semi-extensif ; les ovins profitent de pâturages offerts par des grands parcours à plantes steppiques, dont l'armoise blanche (Artemisia herba-alba), l'alfa (Stipa tenacissima), le diss (Ampelodesmos tenax) et le sennagh (Lygeum spartum), ainsi que par des prairies annuelles, composées de diverses graminées, crucifères et légumineuses. En été, les animaux paissent sur les chaumes de céréales, mais en hiver l'apport en supplément alimentaire est rarement assuré.

Les animaux sont laissés à l'extérieur pendant la journée durant la grande partie de l'année. En hiver, ils ne sont gardés complètement en bergerie que pour une période limitée, selon les conditions météorologiques.

Des traitements anthelminthiques de groupe, sans distinction entre jeunes et adultes, sont effectués généralement en début de printemps et d'automne. Néanmoins, certains éleveurs ne font réaliser aucun traitement, en raison du coût des produits.

La race Ouled Djellal est dessaisonnée ; les béliers sont en permanence dans les troupeaux et la lutte est libre. Les agnelages ont lieu en majorité en plein automne mais débutent dès septembre. Les jeunes et les adultes utilisent les mêmes pâturages.

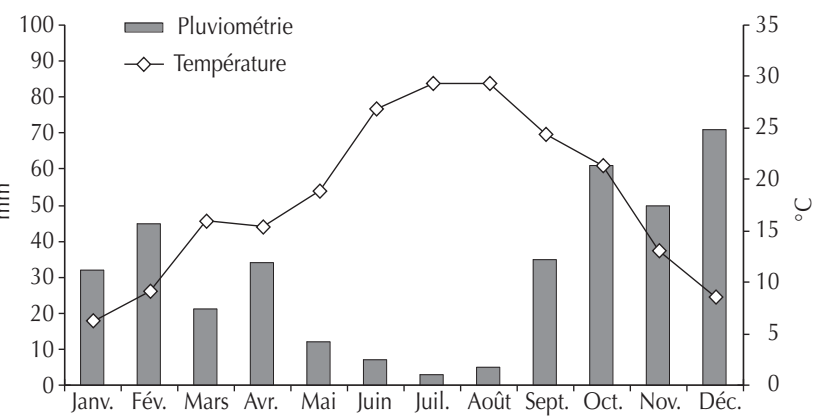

Figure 1 : données climatiques (pluviométrie, température) de la wilaya de Tiaret (Algérie) relatives à l'année 2004 (station météorologique de Ain Bouchekif, Tiaret).

\section{Etude du parasitisme}

Le manque de collaboration de la plupart des éleveurs qui ne souhaitaient pas que leur cheptel soit contrôlé plusieurs fois dans l'année (bien qu'il leur ait été proposé un traitement anthelminthique gratuit) a conduit les auteurs à visiter une seule fois un total de 32 fermes privées, réparties sur les différents points cardinaux de la région.

Les sujets, appartenant aux deux sexes, ont été choisis au hasard parmi des animaux âgés de cinq mois et plus, infestés naturellement au pâturage et non traités aux anthelminthiques depuis huit semaines. Les prélèvements de fèces ont été réalisés au cours de toute l'année 2004, une à deux fois par semaine, sur une dizaine d'individus à la fois, à l'aide de gants en plastique dans le rectum de chaque animal.

L'analyse coprologique a été réalisée au laboratoire de parasitologie de l'université de Tiaret le jour même des prélèvements. Les méthodes utilisées ont été les suivantes : la technique qualitative par flottaison en tube au moyen d'une solution saline saturée, de densité 1,2 ; la technique quantitative à l'aide d'une lame McMaster à deux chambres (Verrerie Dumas, Noisay, France), au seuil de 50 œufs/gramme de fèces (opg) et la coproculture en boîte de Petri à $24{ }^{\circ} \mathrm{C}$ pendant 10 jours (37).

La technique de flottaison utilisée pour la détection des cas positifs permet de retrouver des nématodes (œufs et larves), des cestodes (œufs) et des coccidies (oocystes) $(17,23)$, identifiés par leurs caractères morphologiques respectifs. Dans le cas des nématodes, l'identification des larves L3 obtenues par coproculture a été basée sur les clefs de Niec (29), RVC-FAO (33) et Audebert et coll. (3), pour Nematodirus spp. en particulier. L'observation a été réalisée à l'aide d'un microscope doté d'un objectif à micromètre étalonné.

\section{Analyse des données}

En vue de l'étude parasitologique, l'échantillon de moutons contrôlés a été séparé en deux classes d'âge : les jeunes, d'un âge inférieur à un an, et les adultes.

Les calculs statistiques (écart-type, comparaison de moyennes et de pourcentages, test $t$ et corrélation) ont été effectués à l'aide du logiciel Microsoft Excel (vers. 2000) et du logiciel Statistica (vers. 1997, StatSoft, Tulsa, OK, USA) ( $<<0,05)$.

\section{RESULTATS}

Le nombre total de moutons contrôlés a été de 423 têtes, composé de 19 béliers, 240 brebis et 164 jeunes. L'effectif de moutons parasités a été de 334 , donnant un taux global d'infestation de 78,9 p. 100, pour les parasites étudiés. Les béliers, en raison de leur faible effectif, ont été exclus du reste de l'étude.

Le parasitisme a eu un net caractère saisonnier. Il a été multiple, partagé entre des coccidies (Eimeria spp.), des cestodes (Moniezia expansa, Moniezia benedeni, Avitellina centripunctata), des nématodes du tube digestif (Marshallagia marshalli, Nematodirus spathiger, Nematodirus filicolis, Nematodirus battus, Teladorsagia sp., Trichostrongylus sp., Haemonchus sp., Cooperia sp., Bunostomum sp., Chabertia sp., Trichuris ovis), et des nématodes de l'appareil respiratoire (Dictyocaulus filaria, Protostrongylus rufescens, Muellerius capillaris).

La prévalence globale a été de 9,2 p. 100 pour les nématodes de l'appareil respiratoire, dominés par D. filaria. Ce taux a dû être sous-estimé du fait de la moindre efficacité de la technique de flottaison par rapport à celle de Baermann. Les prévalences des autres 
parasites chez les brebis et les jeunes ovins sont présentées dans le tableau I.

Les nématodes du tube digestif ont été essentiellement des strongles. Des coprocultures de 14 échantillons des mois d'octobre et de novembre ont permis d'identifier, après comptage de 50 larves par échantillon : Teladorsagia et Marshallagia sp. (29 p. 100), Chabertia et Oesophagostomum sp. (21 p. 100), Haemonchus sp. (16 p. 100), Trichostrongylus sp. (15 p. 100), Bunostomum sp. (13 p. 100) et Cooperia sp. (6 p. 100). Cependant, à cause de la ressemblance des œufs de ces différents nématodes à l'examen coproscopique, les auteurs les ont désignés sous le terme de « autres strongles digestifs » (asd) par rapport aux genres Nematodirus, Marshallagia et Trichuris.

Les asd ont été dominants dans les deux classes d'âge des ovins (tableau I); seules des coprocultures quantitatives mensuelles et des autopsies à l'abattoir permettraient de déterminer de façon plus précise les espèces dominantes. Les œufs de $N$. battus (sept cas seulement) ont été observés irrégulièrement entre septembre et février.

Le taux d'infestation des brebis par les strongles (correspondant à celui des nématodes digestifs) a présenté deux pics, le premier en mars (77,7 p. 100), le second, relativement plus prononcé, en novembre (96,6 p. 100) (figure 2). L'effectif des jeunes ovins contrôlés a été assez faible à certaines périodes ( $<10$ sujets/mois) pour permettre une réelle appréciation de l'évolution du parasitisme ; néanmoins, deux pics ont également été relevés (figure 3).

La morphologie caractéristique des œufs des strongles des genres Marshallagia et Nematodirus a permis d'en suivre le parasitisme par rapport à celui des asd (figure 4) chez les brebis dont l'effectif a été le plus élevé.

La figure 5 montre en détail l'évolution des opg des strongles digestifs au cours de l'année 2004. C'est au cours du mois de novembre que l'excrétion fécale des œufs a été la plus élevée, avec un maximum individuel de 7250 opg chez une brebis, tous nématodes confondus. Au niveau spécifique, les valeurs maximales ont été observées en novembre pour $N$. spathiger (450 opg), M. marshalli (400 opg) et les asd (6000 opg), mais en décembre pour T. ovis (1 650 opg).

Sur les 257 ovins parasités par des nématodes, la prévalence des cas d'opg individuels inférieurs à 500 a été de 74,1 p. 100 , celle des opg individuels supérieurs ou égaux à 1000 (seuil généralement admis pour les infestations importantes) n'a été que de 14 p. 100 et la moyenne mensuelle d'opg n'a dépassé ce seuil qu'en novembre chez les deux classes d'âges considérées.

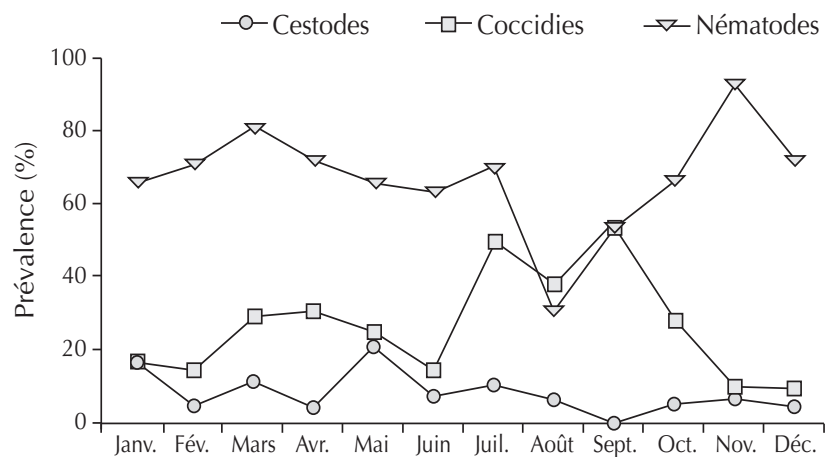

Figure 2 : évolution de l'infestation des brebis de race Ouled Djellal (âge > 1 an) par des parasites internes pendant l'année 2004 dans la région de Tiaret (Algérie). Taux des prévalences mensuelles relatives aux coccidies, cestodes et nématodes sur un effectif de 240 sujets contrôlés.

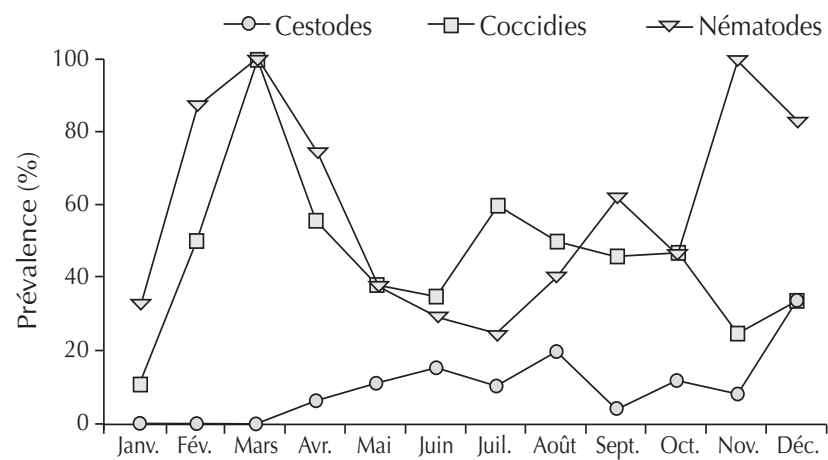

Figure 3 : évolution de l'infestation des jeunes ovins de race Ouled Djellal (âge $<1$ an) par des parasites internes pendant l'année 2004 dans la région de Tiaret (Algérie). Taux des prévalences mensuelles relatives aux coccidies, cestodes et nématodes sur un effectif de 164 sujets contrôlés.

\section{Tableau}

Prévalences des parasites gastro-intestinaux identifiés chez les brebis ( $>1$ an) et les jeunes ovins (5 à 12 mois) de race Ouled Djellal de la région de Tiaret (Algérie) au cours de I'année 2004

\begin{tabular}{|c|c|c|c|c|c|c|c|}
\hline \multirow[b]{4}{*}{$\begin{array}{l}\text { Brebis } \\
(\mathrm{n}=240)\end{array}$} & \multicolumn{7}{|c|}{ Prévalences (\%) } \\
\hline & \multirow[t]{2}{*}{ Coccidies } & \multirow[t]{2}{*}{ Cestodes } & \multicolumn{5}{|c|}{ Nématodes } \\
\hline & & & Nematodirus spp. & $\begin{array}{c}\text { Marshallagia } \\
\text { marshalli }\end{array}$ & Trichuris ovis & ASD & $\begin{array}{c}\text { Tous strongles } \\
\text { digestifs }\end{array}$ \\
\hline & 24,1 & 8,3 & 27 & 28,3 & 5,8 & 56,6 & 70,4 \\
\hline $\begin{array}{l}\text { Jeunes } \\
(n=164)\end{array}$ & 44,5 & 9,7 & 25,6 & 21,3 & 6 & 42 & 53,6 \\
\hline
\end{tabular}




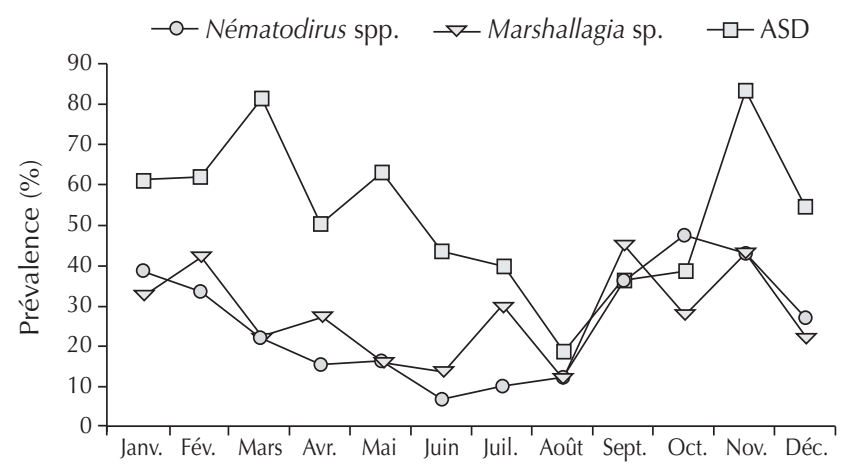

Figure 4 : évolution de l'infestation des brebis de race Ouled Djellal (âge $>1$ an) par les strongles digestifs pendant l'année 2004 dans la région de Tiaret (Algérie). Taux de leurs prévalences relatives sur un effectif de 240 sujets contrôlés. $A S D=$ autres strongles digestifs.

L'infestation par les cestodes adultes a été faible dans les deux classes d'âge (8,3 - 9,7 p. 100), mais plus durable chez les brebis (figure 2). Parmi les deux espèces dominantes, Moniezia expansa a été environ trois fois plus fréquente que $M$. benedeni.

Chez les jeunes, l'infestation par les coccidies a été importante (44,5 p. 100), avec une élévation apparente en début de printemps (figure 3). Les espèces n'ont pas été identifiées.

\section{DISCUSSION}

Le taux d'infestation globale de 78,9 p. 100 a reflété une certaine importance du parasitisme interne des ovins de race Ouled Djellal dans la région de Tiaret. Il s'agissait d'un polyparasitisme à évolution saisonnière.

Les cestodes adultes ont été peu représentés dans les deux classes d'âge des ovins par rapport aux nématodes. Dans l'étude de Bendine et Zahor (6), basée sur des autopsies et des coproscopies, effectuée dans la même région sur 133 moutons, mais limitée à trois mois, leur prévalence a été de 20 p. 100, où seul le genre Moniezia a été cité. Cette faible prévalence devait être en relation avec le cycle indirect et la courte longévité des parasites (37) qui diminuent les possibilités de réinfestation, mais aussi avec la nature de l'échantillon étudié ici (âge $\geqslant 5$ mois), l'immunité acquise avec ce dernier étant un facteur limitant. Ceci pourrait expliquer aussi l'absence de Strongyloides papillosus dans l'échantillon étudié (14).

En revanche, le parasitisme coccidien, non étudié auparavant dans la région, a été relativement important chez les jeunes (44,5 p. 100) et non négligeable chez les brebis (24,1 p. 100). Les coccidies ont un cycle direct et leurs oocystes sont très résistants dans le milieu après sporulation à la faveur d'une température et d'un taux d'humidité élevés (14). La contamination est facilement assurée chez des animaux confinés dans des bergeries mal entretenues, comme c'était le cas dans la présente étude. Ces parasites ont dû jouer un rôle dans les nombreux cas de mortalité observés chez les agneaux dans la région suite à des syndromes diarrhéiques (25), d'où la nécessité de traitements adéquats.

Le taux d'infestation par les nématodes, dont la grande part revenait aux strongles digestifs, n'a pas été très élevé $(53,6$ p. 100 chez les jeunes et 70,4 p. 100 chez les brebis). Dans l'étude de Bendine et Zahor (6), il a été de 70 p. 100. Dans certaines régions

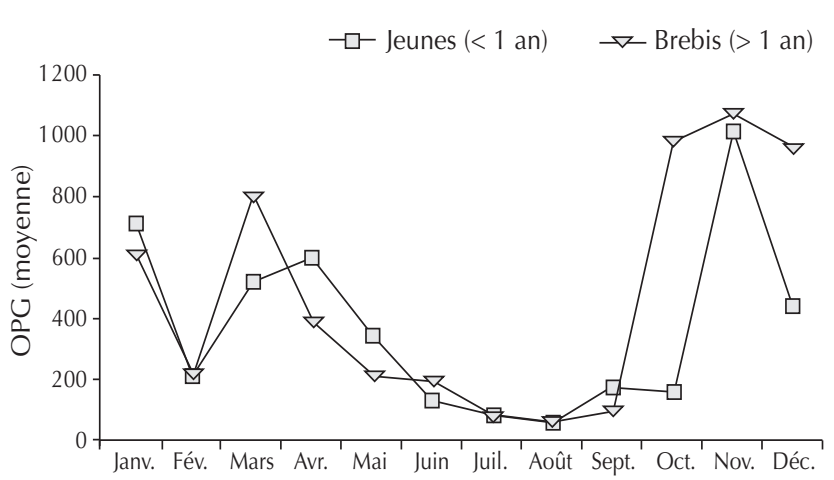

Figure 5 : évolution des niveaux moyens d'excrétion fécale des œufs (opg) des strongles digestifs chez les brebis $(n=240)$ et les jeunes ovins $(n=164)$ de race Ouled Djellal de la région de Tiaret (Algérie), infestés naturellement pendant l'année 2004.

d'Afrique, il peut atteindre 89 à 100 p. 100 des moutons $(1,5,31)$. Le présent échantillon de moutons comprenait peu de sujets très jeunes, plus prédisposés au parasitisme.

L'infestation a été caractérisée par deux pics, avec une certaine recrudescence à partir de l'automne. Cet état devait être dû à un effet cumulatif en rapport avec une infestation précoce en fin d'hiver et de nouvelles contaminations au cours du printemps, en particulier chez les brebis et les agneaux. Dans la région de Tiaret les mises bas ont lieu au printemps et surtout en automne. L'exposition des ovins, favorisée par les conditions climatiques, est alors maximale. Cette situation est retrouvée par ailleurs au Maroc (32).

La coprologie quantitative est considérée fiable, sa corrélation significative avec le degré d'infestation des ovins par des strongles digestifs a été rapportée (11). Dans la région de Tiaret, les moyennes d'excrétion fécale mensuelles maximales ont été de 1081 opg chez les brebis et de 1020 opg chez les jeunes, assez faibles comparées au taux dépassant 8600 opg dans l'étude d'Abebe et Esayas (1) dans une zone semi-aride d'Ethiopie. Sur toute l'année, les opg de tous les ovins infestés ont été 1,6 fois moins élevés que ceux relevés dans la région de Rabat au Maroc (32).

Comme les taux d'infestation, ceux des opg ont été les plus élevés en période froide ; l'indice a montré, chez les brebis comme chez les jeunes, une évolution inversement corrélée avec la température $(\mathrm{r}=-0,69 \mathrm{à}-0,8, \mathrm{p}<0,05)$, mais positivement corrélée avec la pluviométrie $(\mathrm{r}=0,34$ à $0,62, \mathrm{p}<0,05)$, situation relevée aussi dans d'autres régions d'Afrique (31). C'est donc cette période qui devait être la plus favorable au parasitisme. Par ailleurs, les ovins étaient alors très affaiblis à cause de la disette de la saison précédente et le phénomène d'augmentation des opg lors du péripartum devait intervenir fortement chez les brebis $(8,13)$.

Les valeurs d'opg varient en fonction de la fertilité des femelles de strongles : 250 œufs/jour chez Nematodirus sp., et plus de 10000 œufs/jour chez Haemonchus, par exemple (Veterinary Parasitology Vpth603, Laboratory manual 2005, School of Veterinary Medicine, University of Pennsylvania, PA, USA). Dans les infestations ovines mixtes, Kingbury (24) et Tarazona (36) considèrent un taux individuel d'excrétion de 2000 opg comme moyen. La faible fréquence des taux individuels d'opg supérieurs ou égaux à 1000 observée au cours de l'année (14 p. 100 des cas positifs) permet donc de déduire que les infestations strongyliennes chez les ovins ont été modérées dans la région et qu'il était inutile de les déparasiter dans 
certains cas (opg < 500) (38). Cette situation devait être fonction, entre autres, des conditions climatiques rigoureuses qui ont un effet limitant sur le développement des stades larvaires dans le milieu. Ainsi, à partir de mai jusqu'en septembre, puis en février, les opg ont été significativement faibles.

Malgré ce que montre la figure 5, la différence des moyennes d'opg entre novembre $(1081 \pm 1547)$ et mars $(811 \pm 666)$ chez les brebis n'a pas été significative ( $\mathrm{p}>0,05)$. Il existe plusieurs facteurs qui peuvent faire varier les intensités d'excrétion fécale au niveau individuel (la période, le lieu, l'état sanitaire de l'animal, la conduite de l'élevage).

Le manque d'homogénéité des âges des ovins contrôlés n'a pas permis de déterminer de façon significative les catégories ayant le plus fort taux d'excrétion fécale des œufs de strongles $(10,20)$. C'est pendant le pic de novembre que les opg spécifiques maximaux ont été relevés, sauf chez T. ovis (en décembre). Les conditions écologiques de la région devaient être peu favorables à cette espèce qui n'a été identifiée que chez 5,8 à 6 p. 100 des ovins.

Bendine et Zahor (6) ont obtenu pour cette espèce, après analyse coprologique par la méthode de sédimentation, une prévalence de 11,6 p. 100. Les infestations devaient être rares à cause de son cycle évolutif qui peut durer jusqu'à deux mois (17) et l'exigence d'une importante humidité pour les œufs (14), tout en tenant compte de la rareté de ces parasites eux-mêmes dans le gros intestin.

Les opg individuels qui ont atteint un maximum de 6000 parmi les asd devaient être probablement liés à la présence de strongles à prolificité élevée tels que Haemonchus, ou Chabertia et Oesophagostomum (12), comme l'ont révélé les coprocultures d'octobre et de novembre.

L'infestation des brebis par M. marshalli et Nematodirus spp. a été très similaire, calquée sur celle des autres strongles, mais à des taux mensuels toujours plus faibles. Il y a eu une certaine corrélation entre eux : $r=0,66$ chez les brebis, et $r=0,75$ chez les jeunes, avec $p<0,05$. Ils devaient donc avoir les mêmes caractères évolutifs dans la région, malgré le développement exogène plus long des larves chez Nematodirus (37). Dans l'étude de Bendine et Zahor (6), leurs prévalences respectives ont été de 30 et 48 p. 100, relativement proches des présents résultats.

L'infestation des jeunes par M. marshalli a semblé plus faible que chez les brebis, mais statistiquement il n'y a pas eu de différence significative.

Malgré la prévalence modérée et les faibles taux d'excrétion fécale des œufs chez Nematodirus spp. par rapport aux autres strongles, ces parasites doivent avoir des charges importantes chez les animaux. Ainsi Niven et coll. (30) ont trouvé qu'un taux de 200 opg correspondait à une charge de 6000 vers. Ils doivent être considérés comme dangereux, notamment pour les agneaux chez lesquels leur pathogénicité peut être forte $(10,38)$.

Chez les brebis, l'infestation par les strongles a régressé à partir de décembre jusqu'en février, le froid hivernal limitant le déplacement des animaux, qui sont gardés en bergerie, et entraînant des pertes chez les parasites aux différents stades exogènes (œufs, larves). De même, chez les sujets déjà parasités, il devait y avoir une baisse de la fertilité chez les femelles de strongles et une éventuelle hypobiose (de faible ampleur et de courte durée), qui dépend en partie de la température ambiante subie par les larves (9). L'hypobiose permettrait d'expliquer la remontée subite des taux d'infestation et des opg au mois de mars, où la température redevient douce.

La chute des opg à partir d'avril dans le cas des brebis a paru très précoce, au vu des conditions climatiques encore favorables au parasitisme. Cela pouvait être en rapport avec des traitements antérieurs aux avermectines, de plus en plus utilisées en raison de leur large spectre et leur rémanence assez importante (35), ou bien avec un meilleur état de protection immunitaire chez les animaux (34) qui pâturent des pâturages de bonne qualité en cette période.

La chute du parasitisme strongylien en été dans les deux classes d'âge des ovins, malgré la baisse de leur état de santé par malnutrition et sa reprise d'automne, suggère une hypobiose estivale chez la plupart des strongles, comme cela a été déjà décrit ailleurs pour la saison $(18,21,32)$.

Il a été ainsi possible de déterminer de façon plus précise les moments les plus critiques de l'année relatifs au parasitisme interne des ovins de la région, où les traitements anthelminthiques étaient surtout effectués au cours du printemps. Contre les strongles, il est donc suggéré deux déparasitages préventifs par an, au début des mois de mars et d'octobre, à partir d'un certain niveau d'infestation (opg > 500) afin de contrôler le parasitisme et d'éviter le développement d'une résistance chez les parasites (20). Selon le principe des traitements dits ciblés (20) qui sont plus économiques, des traitements anthelminthiques peuvent être recommandés chez les brebis vers la fin de la gravidité, et chez les agneaux après le sevrage, l'immunité contre les strongles se développant par la suite $(14,37,38)$.

Des études ont relevé l'influence de certaines plantes sur l'infestation des animaux par les strongles ou sur la fertilité de ces derniers $(2,19,22)$, ainsi qu'une résistance naturelle aux nématodes chez certaines races ovines $(34,39)$. Outre les conditions climatiques de la région, certaines plantes steppiques, qui constituent une base importante de l'alimentation du cheptel sur les parcours, ou des aptitudes génétiques chez la race Ouled Djellal pourraient jouer un rôle dans la relative limitation du parasitisme strongylien, méritant que des études soient entreprises à leur sujet.

\section{CONCLUSION}

L'étude a révélé que le parasitisme interne des ovins de race Ouled Djellal en région semi-aride d'Algérie était permanent et multiple. Il avait une évolution saisonnière marquée et était dominé par des strongles digestifs, parmi lesquels étaient souvent présents ceux des genres Marshallagia et Nematodirus. La présence fréquente de coccidies et, à moindre degré, de cestodes adultes, surtout en saison chaude, induit une adaptation des traitements prophylactiques, notamment chez les agneaux.

La coprologie quantitative a montré sont intérêt et aurait permis d'éviter des traitements inutiles, le niveau d'excrétion fécale des œufs de strongles ayant été très inférieur à 1000 opg dans de nombreux cas. Elle a révélé que le parasitisme était important à partir de l'automne, alors que les traitements étaient plus fréquemment effectués au printemps.

Bien que la majorité des ovins aient été parasités, les opg globalement peu importants au cours de l'année ont indiqué que les ovins étaient généralement en équilibre avec les strongles, à la faveur des conditions géoclimatiques et/ou zootechniques de la région. Cependant, ils sont demeurés exposés à un déséquilibre en faveur des parasites à certaines occasions (période de disette ou de mise bas). Des traitements saisonniers plutôt individuels devraient donc être entrepris avant les pics observés chez les brebis gravides et les agneaux après le sevrage pour une meilleure efficacité. Les rôles éventuels de la végétation autochtone et de la race ovine dans la limitation du parasitisme, du moins relatif aux strongles, seraient intéressants à évaluer. 


\section{Remerciements}

Nous remercions tous ceux qui ont participé à la réalisation de ce travail, en particulier Dr S. Aïssat, vétérinaire inspecteur, Dr A. Aït Amar, vétérinaire privé, et M. B. Azzouzi du département des Sciences agronomiques de l'université de Tiaret. Nous remercions également Dr J. Cabaret et Dr D. Kerbœuf pour leur accueil à l'unité Bioagresseurs de l'Inra-Nouzilly, France, et leur aide dans la diagnose des strongles.

\section{BIBLIOGRAPHIE}

1. ABEBE W., ESAYAS G., 2001. Survey of ovine and caprine gastrointestinal helminthosis in eastern part of Ethiopia during the dry season of the year. Revue Méd. vét., 152: 379-384.

2. AKROUT A., 2004. Etude des huiles essentielles de quelques plantes pastorales de la région de Matmata (Tunisie). In : Ferchichi A., Réhabilitation des pâturages et des parcours en milieux méditerranéens. Paris, France, Ciheam-lamz, p. 289-292.

3. AUDEBERT F., CASSONE J., KERBOEUF D., DURETTE-DESSET M.C., 2004. Development of Nematodirus spathiger (Nematoda, Molineoidea) in the rabbit and comparison with other Nematodirus spp. parasites of ruminants. Parasitol. Res., 94: 112-117.

4. BARGER I.A., MILLER J.E., KLEI T.R., 1999. The role of epidemiological knowledge and grazing management for helminth control in small ruminants. Int. J. Parasitol., 29: 41-47.

5. BASTIAENSEN P., DORNY P., BATAWUI K., BOUKAYA A., NAPALA A., HENDRICKX G., 2003. Parasitisme des petits ruminants dans la zone périurbaine de Sokodé, Togo. I. Ovins. Revue Elev. Méd. vét. Pays trop., $56: 43-50$.

6. BENDINE T., ZAHOR Z., 1988. Les infestations parasitaires chez les petits ruminants dans la région de Tiaret. Mémoire TSHA, université de Tiaret, Algérie, $60 \mathrm{p}$.

7. BOULKABOUL A., BOUAKKAZ A., KERBOEUF D., 2006. Détection d'une résistance aux benzimidazoles chez les strongles digestifs du cheval en Algérie. Revue Méd. vét., 157 : 59-64.

8. BRUNSDON R.V., 1970. The spring-rise phenomenon: seasonal changes in the worm burdens of breeding ewes and in the availability of pasture infection. N.Z. Vet. J., 18: 47-54.

9. CABARET J., 1977. Inhibition du développement larvaire des strongles gastro-intestinaux des ruminants domestiques, conséquences épidémiologiques. Rec. Méd. vét., 153 : 419-427.

10. CABARET J., 2004. Parasitisme helminthique en élevage biologique ovin : réalités et moyens de contrôle. Prod. Anim., 17 : 145-154.

11. CABARET J., GASNIER N., JACQUIET P., 1998. Faecal egg counts are representative of digestive-tract strongyle worm burdens in sheep and goats. Parasite, 5: 137-142.

12. CABARET J., OUHELLI H., 1984. Fertilité des strongles parasites du tube digestif des ovins dans les conditions naturelles. Revue Méd. vét., $135: 627-633$.

13. CHARTIER C., HOSTE H., BOUQUET W., MALPAUX B., PORS I. $\mathrm{KOCH}$ C., 1998. Periparturient rise in fecal egg counts associated with prolactin concentration increase in French Alpine dairy goats. Parasitol. Res., 84: 806-810.

14. CHARTIER C., ITARD J., MOREL P.-C., TRONCY P.-M., 2000. Précis de parasitologie vétérinaire tropicale. Paris, France, Editions Tec. \& Doc. Lavoisier, $773 \mathrm{p}$.

15. CHELLIG R., 1992. Les races ovines algériennes. Alger, Algérie, Office des publications universitaires, $80 \mathrm{p}$.

16. COOP R.L., HOLMES P.H., 1996. Nutrition and parasite interaction. Int. J. Parasitol., 26: 951-962.

17. FOREYT W.J., 2001. Veterinary parasitology. Reference manual, 5th Edn. Ames, IA, USA, lowa State University Press, 235 p.

18. GIBBS H.A., 1986. Hypobiosis in parasitic nematodes - an update. Adv. Parasitol., 25: 129-174.
19. GITHIORI J.B., 2004. Evaluation of anthelmintic properties of ethnoveterinary plant preparations used as livestock dewormers by pastoralists and small holder farmers in Kenya. Doct. thesis, Swedish University of Agricultural Sciences, Uppsala, Sweden, 72 p.

20. HOSTE H., CHARTIER C., LE FRILEUX Y., 2002. Control of gastrointestinal parasitism with nematodes in dairy goats by treating the host category at risk. Vet. Res., 33: 531-545.

21. HOSTE H., LE FRILEUX Y., POMMARET A., GRUNER L., VAN QUACKEBEKE E., KOCH C., 1999. Importance du parasitisme par des strongles gastro-intestinaux chez les chèvres laitières dans le Sud-Est de la France. Prod. Anim., 12 : 377-389.

22. IDRIS U.E., ADAM S.E., TARTOUR G., 1982. The anthelmintic efficacy of Artemisia herba alba against Haemonchus contortus infection in goats. Natl Inst. Anim. Health Q. (Tokyo), 22: 138-143.

23. KAUFMANN J., 1996. Parasitic infections of domestic animals. A diagnostic manual. Basel, Switzerland, Birkhäuser Verlag, 423 p.

24. KINGBURY P.A., 1965. Relationship between egg counts and worm burdens of young sheep. Vet. Rec., 77: 900-901.

25. KORNI R., 2004. Les pathologies néonatales ovines à Tiaret et Relizane. Mémoire Doct. vét., université Ibn Khaldoun, Tiaret, Algérie, $55 \mathrm{p}$.

26. LLOYD S., SOULSBY E.J.L., 1998. Is anthelmintic resistance inevitable? Back to basics. Equine vet. J., 30: 280-283.

27. MCLEOD R.S., 1995. Cost of major parasites to the Australian livestock industries. Int. J. Parasitol., 25: 1363-1367.

28. MEKHANCHA F., 1988. Etude bibliographique de la taxonomie des helminthes parasites des ruminants domestiques existant en Algérie. Mémoire Doct. vét., ISV, Constantine, Algérie, 89 p.

29. NIEC R., 1968. Cultivo e identificacion de larvas infectantes de nematodes gastrointestinales del bovino y ovino. Manual técnico $\mathrm{n}^{\circ} 3$. Buenos Aires, Argentina, INTA, 37 p.

30. NIVEN P., ANDERSON N., VIZARD A.L., 2002. Trichostrongylid infections in sheep after rainfall during summer in southern Australia. Aust. Vet. J., 80: 567-570.

31. OUATTARA L., DORCHIES P.H., 2001. Helminthes gastro-intestinaux des moutons et chèvres en zones sub-humide et sahélienne du Burkina Faso. Revue Méd. vét., 152 : 165-170.

32. PANDEY V.S., OUHELLI H., DAKKAK A., CABARET J., 1990. Epidemiology of gastrointestinal helminths of sheep in the Rabat area of Morocco. Ann. Rech. vét., 21: 259-266.

33. RVC-FAO, 2004. The RVC/FAO guide to veterinary diagnostic parasitology. Part 1 Ruminants. http://www.fao.org/ag/againfo/resources/ documents/Parasitology/RuminantL3/Introduction.htm. Consulté le $08 / 11 / 2004$.

34. STEAR M.J., WAKELIN D., 1998. Genetic resistance to parasite infection. Revue sci. tech. Off. int. Epizoot., 17: 143-153.

35. SUAREZ V.H., 2002. Helminthic control on grazing ruminants and environmental risks in South America. Vet. Res., 33: 563-573.

36. TARAZONA J.M., 1986. A method for the interpretation of parasite egg counts. Vet. Parasitol., 22: 113-119.

37. URQUHART G.M., ARMOUR J., DUNCAN J.L., DUNN A.M., JENNING F.W., 1996. Veterinary parasitology, 2nd Edn. Oxford, UK, Blackwell Science, $292 \mathrm{p}$.

38. VLASSOFF A., LEATHWICK D.M., HEATH A.C.G., 2001. The epidemiology of nematode infections of sheep. N.Z. Vet. J., 49: 213-221.

39. WOOLASTON R.R., BAKER R.L., 1996. Prospects of breeding small ruminants for resistance to internal parasites. Int. J. Parasitol., 26: 845855.

Reçu le 10.01.2006, accepté le 06.03.2007 


\section{Summary}

Boulkaboul A., Moulaye K. Internal Parasites in Algerian Arab Sheep in a Semiarid Area of Algeria

A study of internal parasites was performed on an Algerian Arab sheep breed (Ouled Djellal) naturally infected in a semiarid area of Algeria (Tiaret). Fecal examinations carried out on 423 sheep during the year 2004 revealed a global infection rate of $78.9 \%$ and the presence of cestodes (Moniezia spp., Avitellina sp.), coccidia (Eimeria spp.), and nematodes including lungworms (Dictyocaulus sp., Muellerius sp., Protostrongylus $\mathrm{sp}$.). The parasitism pattern was seasonal for all parasites. Coccidia were more present in lambs $(n=164)$, with a prevalence of $44.5 \%$. The strongyle infection was highest $(70.4 \%)$ in ewes $(n=240)$, with a peak in March and November. The following strongyles were identified: Trichuris ovis (5.8\%), Marshallagia marshalli (28.3\%) and Nematodirus spp. (27\%). The other strongyles, of which some genera/species were identified by fecal culture, had a prevalence of $56.6 \%$. M. marshalli and Nematodirus spp. had a remarkably similar evolution, but at a lower level than that of other strongyles. The fecal egg count (EPG, i.e. eggs/gram of feces) showed two peaks; the highest (average of 1081 EPGs) occurred in November (lambing period) and was probably linked to the periparturient rise. Generally, the strongyle infection was moderate even though it was predominant. The prevalence of individual and under 500 EPGs was $74.1 \%$, that more than or equal to 1000 EPGs was $14 \%$, and the EPG monthly average exceeded this threshold in November only. Besides climatic conditions, the implication of some factors, such as the grazed vegetation type in the area and sheep breed, deserves investigation. For disease control, two anthelmintic treatments (in spring and fall) may be sufficient.

Keywords: Ouled Djellal sheep - Nematoda - Coccidia Cestoda - Feces - Semiarid zone - Algeria.

\section{Resumen}

Boulkaboul A., Moulaye K. Parásitos internos del ovino de raza Ouled Djellal en zona semi árida de Argelia

Se realizó un estudio sobre los parásitos internos en los ovinos de raza Ouled Djellal infestados naturalmente en una región semi árida de Argelia(Tiaret). Los exámenes coprológicos efectuados durante el año 2004 en 423 ovinos revelaron una tasa global de infestación de 78,9\% y permitieron la identificación de céstodos (Moniezia spp., Avitellina sp.), coccidios (Eimeria spp.) y nemátodos, incluyendo estróngilos respiratorios (Dictyocaulus sp., Muellerius sp., Protostrongylus sp.). El parasitismo fue estacional para todos los parásitos. Las coccidiosis fueron las más presentes en los jóvenes $(n=164)$, con una prevalencia de $44,5 \%$. En la oveja $(n=240)$, la infestación por estróngilos digestivos fue la más elevada $(70,4 \%)$, con un pico en marzo y otro en noviembre. Se identificaron los siguientes estróngilos : Trichuris ovis (5,8\%), Marshallagia marshalli $(28,3 \%)$ y Nematodirus spp. (27\%). Los otros estróngilos, de los cuáles algunos géneros/especies fueron identificados por cultivo coprológico presentaron una prevalencia de 56,6\%. M. marshalli y Nematodirus spp. presentaron una evolución notablemente similar, pero de un nivel más bajo que los otros estróngilos. La excreción de los huevos de estróngilos por gramo de heces (opg) presentó dos picos, siendo el más elevado (promedio de 1081 opg) en noviembre (período de partos), ligado probablemente al fenómeno del aumento de opg en el momento del peri parto. Globalmente, el parasitismo ligado a los estróngilos fue moderado, a pesar de su predominancia. La prevalencia de opg individuales inferiores a 500 fue de $74,1 \%$, la de opg superiores o iguales a 1000 fue solamente de $14 \%$ y el promedio mensual de opg sólo sobrepasó este límite en noviembre. Aparte de las condiciones climáticas, la implicación de ciertos factores, tales como la naturaleza de la cobertura vegetal de la región y la raza ovina, ameritan investigación. Para la profilaxis, dos tratamientos anti helmínticos durante el año (primavera y otoño) deberían bastar.

Palabras clave: Ovino Ouled Djellal - Nematoda - Coccidia Cestoda - Heces - Zona semiárida - Argelia. 